\title{
A Simplified Global Solution for an Advection\# dominated Accretion Flow
}

\section{Citation}

Yuan, Feng, Renyi Ma, and Ramesh Narayan. 2008. "A Simplified Global Solution for an Advection\#dominated Accretion Flow." The Astrophysical Journal 679 (2) (June): 984-989. doi:10.1086/587484.

\section{Published Version}

doi:10.1086/587484

\section{Permanent link}

http://nrs.harvard.edu/urn-3:HUL.InstRepos:27814537

\section{Terms of Use}

This article was downloaded from Harvard University's DASH repository, and is made available under the terms and conditions applicable to Other Posted Material, as set forth at http:// nrs.harvard.edu/urn-3:HUL.InstRepos:dash.current.terms-of-use\#LAA

\section{Share Your Story}

The Harvard community has made this article openly available.

Please share how this access benefits you. Submit a story.

\section{Accessibility}




\title{
A SIMPLIFIED GLOBAL SOLUTION FOR AN ADVECTION-DOMINATED ACCRETION FLOW
}

\author{
Feng Yuan, ${ }^{1,2}$ Renyi Ma, ${ }^{1}$ and Ramesh Narayan ${ }^{3}$ \\ Received 2008 January 21; accepted 2008 February 12
}

\begin{abstract}
When we model black hole accretion sources such as active galactic nuclei and black hole X-ray binaries as advectiondominated accretion flows (ADAFs), it is necessary to use the global solution to the equations rather than the simpler selfsimilar solution, since the latter is inaccurate in the region near the black hole where most of the radiation is emitted. However, technically, it is a difficult task to calculate the global solution because of the transonic nature of the flow, which makes it a two-point boundary value problem. In this paper we propose a simplified approach for calculating the global ADAF solution. We replace the radial momentum equation by a simple algebraic relation between the angular velocity of the gas and the Keplerian angular velocity while keeping all other equations unchanged. It is then easy to solve the differential energy equations to obtain an approximate global solution. By adjusting the free parameters, we find that for almost any accretion rate and for $\alpha=0.1-0.3$ we can get good simplified global solutions. The predicted spectra from the approximate solutions are very close to the spectra obtained from the true global solutions.
\end{abstract}

Subject headings: accretion, accretion disks — black hole physics — galaxies: active — galaxies: nuclei — hydrodynamics

\section{INTRODUCTION}

Advection-dominated accretion flow (ADAF) is an important type of solution for black hole accretion. A prominent feature of an ADAF compared to the standard thin disk is its low radiative efficient at low accretion rates (Narayan \& Yi 1994, hereafter NY94; Narayan \& Yi 1995; see Narayan et al. 1998 and Kato et al. 1998 for reviews).

The ADAF solution has received much attention in recent years because it successfully explains why some nearby galaxies are so dim even though their accretion rates are not very small (see Narayan 2005, Yuan 2007, and Ho 2008 for reviews). The best evidence comes from the supermassive black hole in our Galactic center, Sgr A* (Narayan et al. 1995; Manmoto et al. 1997; Yuan et al. 2003). From Chandra observations and Bondi theory, we can estimate the mass accretion rate of $\mathrm{Sgr} \mathrm{A}^{*}$. If the accretion flow were not an ADAF but a standard thin disk, the luminosity would be 5 orders of magnitude larger than that observed (Yuan et al. 2003).

Another attractive feature of an ADAF is that it can partly solve the problem of the origin of X-ray emission from accretion flows. The temperature of a standard thin disk at the inner disk is only $\sim 10^{5} \mathrm{~K}$ for a supermassive black hole, too low to produce X-ray emission (Frank et al. 2002). A hot corona has been thought to be responsible for the X-ray emission, but recent MHD simulations of disks show that they have hardly any coronae (Hirose et al. 2006). On the other hand, the temperature of an ADAF is high enough to produce X-ray emission. Of course, a canonical ADAF exists only below a critical accretion rate $\dot{M}_{\text {crit }} \approx \alpha^{2} \dot{M}_{\text {Edd }}$ with $\dot{M}_{\text {Edd }} \equiv 10 L_{\text {Edd }} / c^{2}$, where $\alpha$ is the viscous parameter, which corresponds to $\sim(3 \%-4 \%) L_{\text {Edd }}$ at most (Esin et al. 1997). Therefore, it cannot explain luminous X-ray sources such as quasars. The luminous hot accretion flow (LHAF; Yuan 2001), which is an extension of an ADAF to higher accretion rates, is promising, but the

\footnotetext{
1 Shanghai Astronomical Observatory, Chinese Academy of Sciences, 80 Nandan Road, Shanghai 200030, China; fyuan@shao.ac.cn.

2 Joint Institute for Galaxy and Cosmology (JOINGC) of SHAO and USTC, China.

${ }^{3}$ Harvard-Smithsonian Center for Astrophysics, 60 Garden Street, Cambridge, MA 02138.
}

details of this model have not been fully worked out (see Yuan et al. 2007 for an example of application to luminous black hole $\mathrm{X}$-ray binaries).

In spite of the great success of ADAFs, more work is required to test the model. On the one hand, it would be useful to expand the application of ADAFs to more sources, and on the another hand, such modeling is expected to help us understand some important microphysical issues that are still unclear. One example of the latter is the value of $\delta$ (defined in eqs. [4] and [5]), which measures the amount of direct electron heating through viscous dissipation in a hot accretion flow (Sharma et al. 2007). Another is the potential importance of collective plasma effects, which will determine how realistic the two-temperature assumption is (Begelman \& Chiueh 1988; Yuan et al. 2006).

For such work, the global solution rather than the self-similar solution of the ADAF equations is required. This is because most of the radiation of an ADAF comes from its innermost region where the self-similar solution breaks down. However, it is technically very difficult to calculate the global solution of an ADAF. An ADAF is transonic, and thus its global solution should satisfy the sonic-point condition in addition to the outer boundary condition. Mathematically, it is a two-point boundary value problem and not easy to deal with. This is an obstacle to the wide application of the ADAF model.

In this paper we propose a simplified global ADAF solution. We adopt a simple algebraic relation to replace the radial momentum equation, thus avoiding the two-point boundary value problem. We present our approach in $\S 2$ and show some examples in $\S 3$. Section 4 is devoted to a short summary. Watarai (2007) has recently presented related work.

\section{THE SIMPLIFIED GLOBAL ADAF MODEL}

The basic equations of an ADAF describe the conservation of mass, radial and aximuthal components of the momentum, and energy (e.g., Narayan et al. 1998):

$$
\begin{gathered}
\dot{M}=-4 \pi r H \rho v \\
v \frac{d v}{d r}=\left(\Omega^{2}-\Omega_{\mathrm{K}}^{2}\right) r-\frac{1}{\rho} \frac{d P}{d r},
\end{gathered}
$$



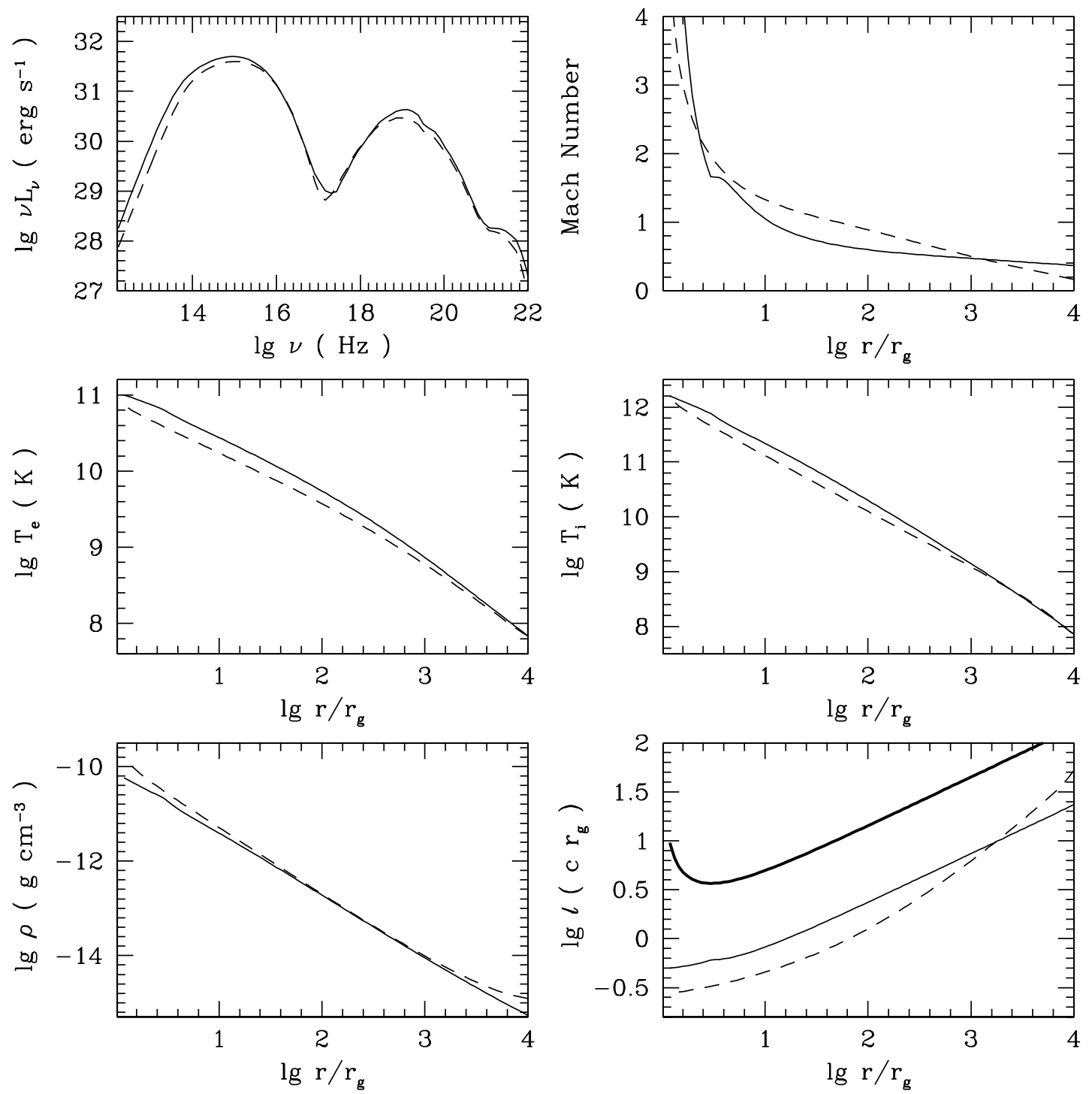

FIg. 1.-Simplified (solid line) and exact (dashed line) global ADAF solutions for $\dot{M}=10^{-5} \dot{M}_{\text {Edd }}, \alpha=0.3$. The parameters are $f_{0}=0.33, j=0.98$.

$$
\begin{gathered}
v\left(\Omega r^{2}-j\right)=-\alpha r c_{s}^{2}, \\
\rho v T_{i} \frac{d s_{i}}{d r}=(1-\delta) q^{+}-q_{i e}, \\
\rho v T_{e} \frac{d s_{e}}{d r}=q_{i e}-q^{-}-\delta q^{+} .
\end{gathered}
$$

All the quantities have their usual meaning. In the present paper we do not include outflows from the ADAF, but it is easy to extend our calculation to that case by simply using a radiusdependent mass accretion rate, $\dot{M}=\dot{M}_{0}\left(r / r_{\text {out }}\right)^{s}$ with $s>0$ being a constant (e.g., Yuan et al. 2003). The quantity $\delta$ in equations (4) and (5) describes the fraction of the turbulent dissipation rate $q^{+}$ that directly heats electrons; we set $\delta=0.3$. The quantity $q_{i e}$ describes the energy transfer rate from ions to electrons by Coulomb collision, and $q^{-}$is the radiative cooling rate. We consider synchrotron and bremsstrahlung emissions and their Comptonization. The details of the calculation of the spectrum can be found in Yuan et al. (2003). We consider a Schwarzschild black hole and adopt the Paczyński \& Wiita (1980) potential to mimic its geometry.

The most difficult part of solving the global solution is the radial momentum equation (2). Our key idea of simplifying the global ADAF solution is to replace this differential equation by the following simple algebraic relation:

$$
\Omega=f \Omega_{\mathrm{K}},
$$

with

$$
\begin{aligned}
& f= \\
& \begin{cases}f_{0} & \text { for } r>r_{\mathrm{ms}}, \\
\frac{f_{0} \Omega_{\mathrm{K}}\left(r_{\mathrm{ms}}\right) r_{\mathrm{ms}}^{2}}{\Omega_{\mathrm{K}} r^{2}}\left(\frac{r}{r_{\mathrm{ms}}}\right)^{n}=f_{0} \frac{r-r_{g}}{2 r_{g}}\left(\frac{r}{r_{\mathrm{ms}}}\right)^{n-3 / 2} & \text { for } r<r_{\mathrm{ms}} .\end{cases}
\end{aligned}
$$

where $r_{\mathrm{ms}}=3 r_{g} \equiv 6 G M / c^{2}$ is the innermost stable circular orbit.

The above simplification is based on the following physical consideration. The immediate idea we think of to simplify the radial momentum equation is to use the self-similar solution obtained by NY94. Consider equations (7)-(9) in NY94. We can use equation (9) in NY94 to solve for $\epsilon^{\prime}$ in terms of the sound speed $c_{s}$ :

$$
5+2 \epsilon^{\prime}=2 \frac{v_{\mathrm{K}}^{2}}{c_{s}^{2}}, \epsilon^{\prime}=\frac{v_{\mathrm{K}}^{2}}{c_{s}^{2}}-\frac{5}{2} .
$$



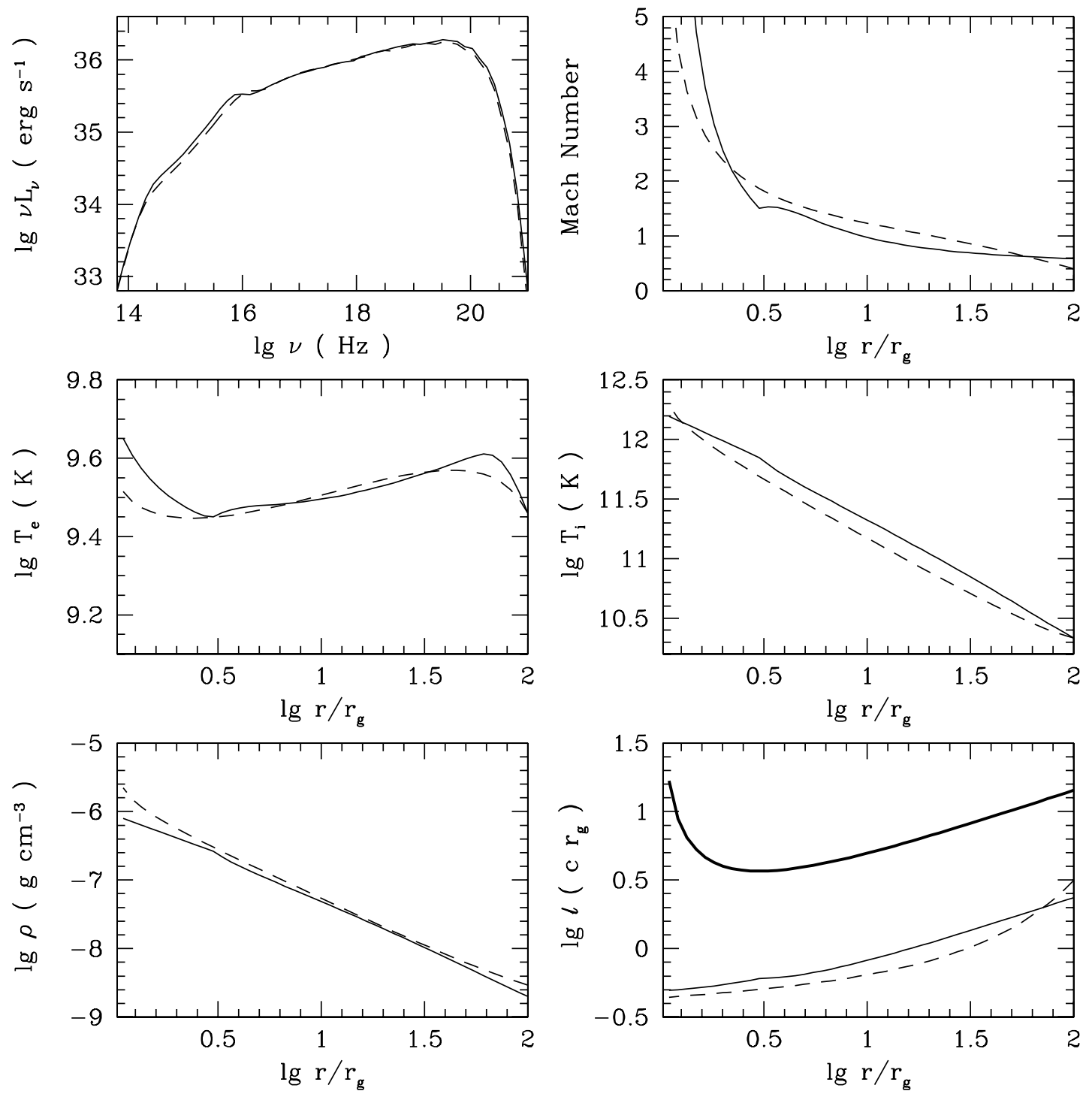

FIG. 2.-Simplified (solid line) and exact (dashed line) global ADAF solutions for $\dot{M}=10^{-1} \dot{M}_{\text {Edd }}, \alpha=0.3$. The parameters are $f_{0}=0.33, j=0.98$.

We can then substitute this in equations (8) and (7) in NY94 to obtain for a fully advection-dominated flow $\left(\epsilon^{\prime}=\epsilon\right)$

$$
\frac{\Omega}{\Omega_{\mathrm{K}}}=\left(1-\frac{5 c_{s}^{2}}{2 v_{\mathrm{K}}^{2}}\right)^{1 / 2}=\frac{10-6 \gamma}{9 \gamma-5}=\mathrm{const},
$$

where $\gamma$ is the adiabatic index. We therefore in principle could set $f$ in equation (6) to this constant. However, we find that the simplified solution is very sensitive to the value of $f$. The reason is that, as we show below, the radial velocity is sensitive to the value of $f$ (see eq. [9] below). The velocity determines the density and also the temperature via the energy equations, two quantities that determine the emitted spectrum. We therefore set $f$ as a free parameter that we adjust for different accretion parameters $\dot{M}$ and $\alpha$ to get the best approximation.

Because the angular momentum $\Omega r^{2}$ in a global solution keeps decreasing with decreasing radius, while the Keplerian angular momentum $\Omega_{\mathrm{K}} r^{2}$ begins to increase when $r<r_{\mathrm{ms}}$ (Fig. 1), $f$ cannot be a constant when $r<r_{\mathrm{ms}}$. Instead we require the angular momentum to be continuous at $r_{\mathrm{ms}}$ and assume that it is proportional to $\left(r / r_{\mathrm{ms}}\right)^{n}$. After some tests we set $n=0.5$, independent of the values of $\dot{M}$ and $\alpha$. Thus, $n$ is not a free parameter in our model.
Substituting equation (6) into equation (3) we have

$$
v_{r}=-\frac{\alpha r c_{s}^{2}}{f \Omega_{\mathrm{K}} r^{2}-j} .
$$

The quantity $j$ is the specific angular momentum of the accretion gas when it falls into the black hole, and it is the eigenvalue of the exact global solution. In our simplified model, we set $j$ as the second free parameter and adjust its value to get the best approximate solution for $v$.

Substituting equations (1), (6), and (9) into the energy equations for ions and electrons, equations (4) and (5), we have two differential equations with two unknown variables, $T_{i}$ and $T_{e}$. All other quantities such as $v, \rho, c_{s}$, and $H$ can be expressed as simple functions of $T_{i}$ and $T_{e}$ for a given $\dot{M}$ and $\alpha$ and assumed values of the free parameters $f_{0}$ and $j$. When $T_{i}$ and $T_{e}$ are given at the outer boundary, we can easily integrate the differential equations inwards to get the approximate global solution.

We adjust the values of $f_{0}$ and $j$ for different $\dot{M}$ and $\alpha$ to get the best simplified global solution. Here "best" means that the profiles of all quantities such as $\rho, v, T_{e}, T_{i}$, and most importantly, the emitted spectrum, are very close to the exact global solution. Because our main purpose is to model the continuum spectrum 

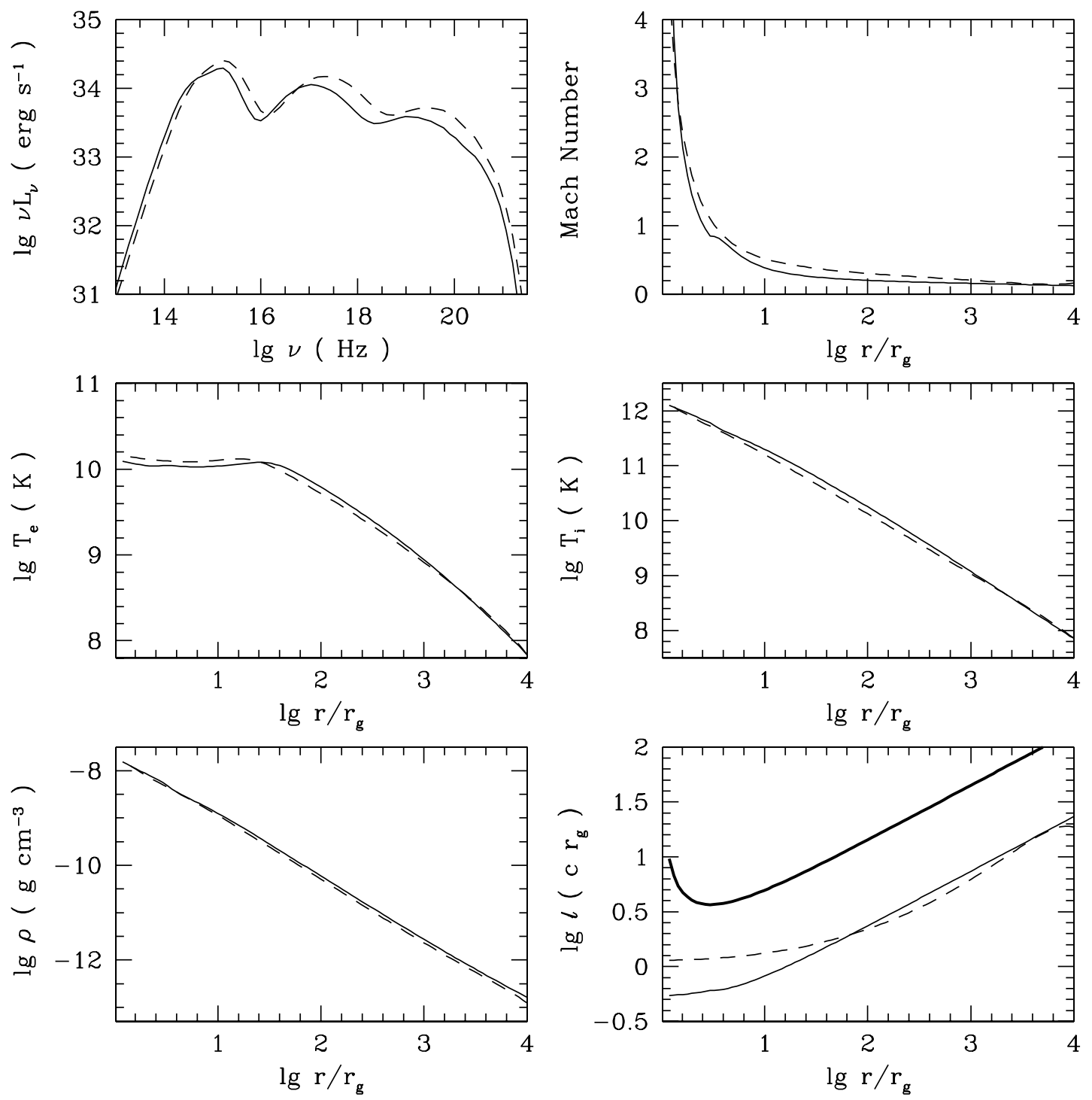

FIg. 3.-Simplified (solid line) and exact (dashed line) global ADAF solutions for $\dot{M}=10^{-3} \dot{M}_{\text {Edd }}, \alpha=0.1$. The parameters are $f_{0}=0.33, j=1.08$.

of black hole sources (AGNs and black hole X-ray binaries), our first priority will be the closeness of the spectrum when we judge how good a simplified solution is.

In the calculations presented here, we set $\dot{M}$ of the approximate solution equal to $\dot{M}$ of the global solution it is meant to fit. However, in real applications, we only know the spectrum rather than $\dot{M}$. So it might be more realistic to adjust $\dot{M}_{\text {simp }}$ of the simplified solution to fit the spectrum produced by the exact global solution with a given $\dot{M}_{\text {exact }}$ rather than setting $\dot{M}_{\text {simp }}=\dot{M}_{\text {exact }}$. Fortunately, we find that $\dot{M}_{\text {simp }}$ and $\dot{M}_{\text {exact }}$ are very close, typically deviating by no more than $\sim 3 \%$.

\section{RESULTS}

When modeling black hole sources with an ADAF model, the accretion rate $\dot{M}$ spans a wide range, say, from $10^{-6} \dot{M}_{\text {Edd }}$ to $10^{-1} \dot{M}_{\text {Edd }}$. But the value of $\alpha$ adopted in ADAF modeling (e.g., Narayan et al. 1998) is usually within a very narrow range, $\alpha=$ $0.1-0.3$. This is also supported by MHD numerical simulations of accretion flows (e.g., Hawley \& Krolik 2001). We adjust the values of $j$ and $f_{0}$ to obtain the "best" simplified ADAF solutions for $\dot{M}$ and $\alpha$ within the above ranges. As stated below we find that the same set of $\left(j, f_{0}\right)$ often holds for quite a wide range of $\dot{M}$.

$$
\text { 3.1. } \alpha=0.3: f_{0}=0.33, j=0.98 \text { for any } \dot{M}
$$

We first present results for $\alpha=0.3$. We find that in this case the simplified global solution with $f_{0}=0.33, j=0.98$ gives a satisfactory spectrum for any $\dot{M}$. Figures 1 and 2 give two examples with $\dot{M}=10^{-5} \dot{M}_{\text {Edd }}$ and $10^{-1} \dot{M}_{\text {Edd }}$, respectively. The dashed lines in the figure denote the exact global solution, while the solid lines are for the simplified global solution. The plots in each figure show the emitted spectrum, Mach number, electron and ion temperatures, density, and the angular momentum, respectively. For Figure 1 the outer boundary is at $10^{4} r_{q}$, and the outer boundary condition is $T_{i}=0.2 T_{\mathrm{vir}}, T_{e}=0.19 T_{\mathrm{vir}}$ with the virial temperature $T_{\text {vir }} \equiv 3.6 \times 10^{12}\left(r_{g} / r\right)$. For Figure 2 the outer boundary is at $10^{2} r_{g}$, and the outer boundary condition is $T_{i}=0.6 T_{\mathrm{vir}}, T_{e}=0.08 T_{\mathrm{vir}}$.

$$
\text { 3.2. } \alpha=0.1: f_{0}=0.33, j=1.08 \text { for } \dot{M} \lesssim 10^{-2} \dot{M}_{\text {Edd }}
$$

When $\alpha=0.1$, it is hard for a single set of $\left(j, f_{0}\right)$ to give a good solution for all $\dot{M}$. When $\dot{M}$ is relatively low, $\dot{M} \lesssim 10^{-2} \dot{M}_{\text {Edd }}$, we 

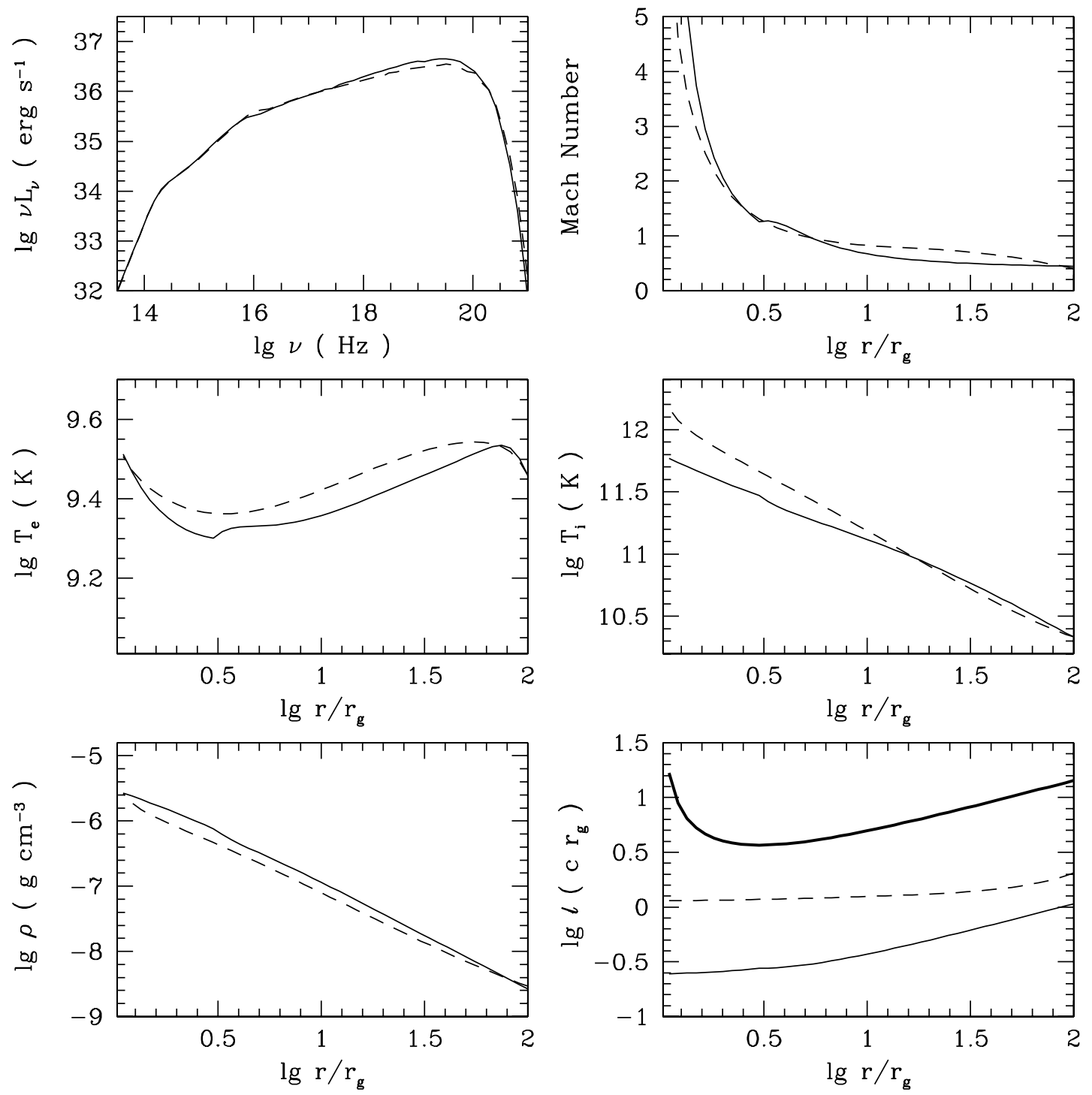

FIG. 4.-Simplified (solid line) and exact (dashed line) global ADAF solutions for $\dot{M}=10^{-1} \dot{M}_{\text {Edd }}, \alpha=0.1$. The parameters are $f_{0}=0.15, j=0.49$.

find $f_{0}=0.33, j=1.08$ gives a satisfactory solution. Figure 3 shows an example with $\dot{M}=10^{-3} \dot{M}_{\text {Edd }}$. The outer boundary conditions are $T_{i}=0.2 T_{\mathrm{vir}}, T_{e}=0.19 T_{\mathrm{vir}}$ at $r_{\text {out }}=10^{4} r_{g}$.

$$
\text { 3.3. } \alpha=0.1: f_{0}=0.15, j=0.49 \text { for } \dot{M} \gtrsim 5 \times 10^{-2} \dot{M}_{\mathrm{Edd}}
$$

When $\dot{M}$ is relatively high, $\dot{M} \gtrsim 5 \times 10^{-2} \dot{M}_{\text {Edd }}$, we find $f_{0}=$ $0.15, j=0.49$ gives a satisfactory solution. Figure 4 shows an example of $\dot{M}=10^{-1} \dot{M}_{\text {Edd }}$. The outer boundary conditions are $T_{i}=0.6 T_{\mathrm{vir}}, T_{e}=0.08 T_{\mathrm{vir}}$ at $r_{\mathrm{out}}=10^{2} r_{g}$. Note that this solution is in the regime of LHAF, because $10^{-1} \dot{M}_{\text {Edd }}$ is well above the critical accretion rate of an ADAF $\dot{M}_{\text {crit }} \approx 10^{-2} \dot{M}_{\text {Edd }}$.

\subsection{Other Values of $\alpha$}

For other values of $\alpha$, we find that simply using the "linear fit" values of $\left(j, f_{0}\right)$ between those for $\alpha=0.3$ and 0.1 gives a good solution. For example, for $\alpha=0.2$ and $\dot{M}=10^{-3} \dot{M}_{\text {Edd }}$, the values of $j$ and $f_{0}$ are just $f_{0}=(0.33+0.33) / 2=0.33, j=(0.98+$ $1.08) / 2=1.03$. For $\alpha=0.2$ and $\dot{M}=0.08 \dot{M}_{\mathrm{Edd}}, f_{0}=(0.33+$ $0.15) / 2=0.24, j=(0.98+0.49) / 2=0.735$.

\section{SUMMARY}

The global solution of ADAFs is difficult to calculate because it is mathematically a two-point boundary value problem. This hampers wide application of the ADAF model. We propose a simplified global solution to overcome this difficulty. Prompted by the self-similar solution of ADAFs, we replace the radial momentum equation, which is the most difficult to handle, with a simple algebraic relation (eq. [6]), and then solve the remaining two differential equations (eqs. [4] and [5]). We adjust the two free parameters ( $j$ and $f_{0}$ in eqs. [6] and [9]) so that we obtain the best approximation compared to the exact global ADAF solution. The spectra of the simplified solutions are impressively good, as shown in Figures $1-4$ for various $\dot{M}$ and $\alpha$.

We have been unable to identify a single set of values of the two adjustable constants $j$ and $f_{0}$, which works for all ADAF models. However, when $\alpha$ is large, say, $\sim 0.3$, we find that $j=0.98$, $f_{0}=0.33$ gives very good results for all accretion rates $\dot{M}$ for which an ADAF solution is possible. Recent work by Sharma et al. (2006) suggests that the viscosity parameter in the collisionless plasma in a hot accretion flow will be larger than in a standard thin disk. Therefore, $\alpha \sim 0.3$ is probably not unrealistic for an ADAF. It would thus be reasonable to use a single set of parameters, $\alpha=0.3, j=0.98, f_{0}=0.33$, for practical applications of the approximate global model described here.

The success of the present work encourages us to extend our approach to the case of a slim disk, which is an extension of the 
standard thin disk to accretion rates above the Eddington rate (Abramowicz 1988). It potentially has important application in ULXs and narrow-line Seyfert 1 galaxies (Mineshige et al. 2000; Watarai et al. 2001). We hope to report the results in a future paper.
This work was supported in part by the Natural Science Foundation of China (grant 10773024), Shanghai Pujiang Program, One-Hundred-Talent Program of CAS (F. Y.), the Knowledge Innovation Program of CAS, and Shanghai Postdoctoral Scientific Program (R. M.).
Abramowicz, M. 1988, ApJ, 332, 646

Begelman, M. C., \& Chiueh, T. 1988, ApJ, 332, 872

Esin, A. A., McClintock, J. E., \& Narayan, R. 1997, ApJ, 489, 865

Frank, J., King, A., \& Raine, D. 2002, Accretion Power in Astrophysics (Cambridge: Cambridge Univ. Press)

Hawley, J. F., \& Krolik, J. H. 2001, ApJ, 548, 348

Hirose, S., Krolik, J. H., \& Stone, J. M. 2006, ApJ, 640, 901

Ho, L. C. 2008, ARA\&A, in press (arXiv: 0803.2268)

Kato, S., Fukue, J., \& Mineshige, S. 1998, Black Hole Accretion Disks (Kyoto: Kyoto Univ. Press)

Manmoto, T., Mineshige, S., \& Kusunose, M. 1997, ApJ, 489, 791

Mineshige, S., et al. 2000, PASJ, 52, 499

Narayan, R. 2005, Ap\&SS, 300, 177

Narayan, R., Mahadevan, R., \& Quataert, E. 1998, in The Theory of Black Hole Accretion Discs, ed. M. A. Abramowicz, G. Bjornsson \& J. E. Pringle (Cambridge: Cambridge Univ. Press), 148

\section{REFERENCES}

Narayan, R., \& Yi, I. 1994, ApJ, 428, L13 1995, ApJ, 452, 710

Narayan, R., Yi, I., \& Mahadevan, R. 1995, Nature, 374, 623

Paczyński, B., \& Wiita, P. J. 1980, A\&A, 88, 23

Sharma, P., et al. 2006, ApJ, 637, 952 2007, ApJ, 667, 714

Watarai, K. Y. 2007, PASJ, 59, 443

Watarai, K. Y., et al. 2001, ApJ, 549, L77

Yuan, F. 2001, MNRAS, 324, 119 2007, in ASP Conf. Ser. 373, The Central Engine of Active Galactic

Nuclei, ed. L. C. Ho \& J.-M. Wang (San Francisco: ASP), 95

Yuan, F., Quataert, E., \& Narayan, R. 2003, ApJ, 598, 301

Yuan, F., et al. 2006, ApJ, 636, 46 2007, ApJ, 659, 541 